\section{Determinants of cataract surgical opportunities and complication rates in UK higher specialist training}

\begin{abstract}
Purpose The volume of cataract surgery performed by trainee ophthalmologists in the UK, and the complication rates experienced by those trainees is unknown. As a result, no appropriate audit benchmark exists for trainees or their trainers. This study describes the surgical opportunities and rates of posterior capsule rupture (PCR) experienced by higher specialist trainees in one UK training region and explores influencing factors.

Methods Number of cataract operations and episodes of PCR in each calendar month were recorded from surgical logbooks for all Specialist Registrars (SpRs) who had completed at least 6 months of training by January 2007. Dates and details of the posts in which the surgery was performed were also recorded.

Results Data from 475 completed months were collected from 19 trainees including 4322 cataract operations and 99 episodes of PCR. Trainees performed a mean 9.1 operations per month. This varied significantly between different subspeciality posts; the fewest cataracts were on paediatric and oculo-plastic firms. District General Hospitals offered more surgery than Teaching Hospitals; mean 10.9 vs 8.5 cataracts per month $(P=0.005)$. No difference in surgical opportunity was found between male and female trainees. An overall PCR rate of $2.3 \%$ (95\% CI 2.25-2.33\%) was found, which decreased significantly with increasing experience $(P<0.01)$.

Conclusion SpRs in Yorkshire expect to complete around 500 cataract operations in their 4.5 years of training. A PCR rate of $2.3 \%$ was found for trainees with a mean of 25 months higher specialist training which
\end{abstract}

JC Buchan and A Cassels-Brown

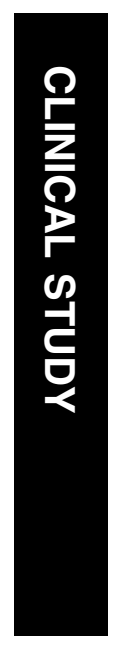

compares favourably with published series of trainees from other countries.

Eye (2008) 22, 1425-1429; doi:10.1038/eye.2008.157; published online 12 September 2008

Keywords: cataract; benchmarking; complications; training

\section{Introduction}

Ophthalmic surgical training in the UK was, until recently, divided into two halves; basic specialist training of around 3 years during which trainees might typically complete 100 cataract extractions (stipulated minimum of 50 completed cases), then a subsequent 4.5 years of 'Specialist Registrar' (SpR) training providing trainees with increasing volumes of cataract surgery and decreasing levels of direct supervision. ${ }^{1}$

Although many factors will influence the process of cataract surgical skill acquisition, the opportunity to undertake sufficient volumes of surgery is a pre-requisite. Previous studies have looked at factors influencing the surgical opportunities of UK trainees in basic specialist training, ${ }^{2,3}$ and while it is stipulated by the Royal College of Ophthalmologists (RCOphth) that SpRs must complete a minimum of 300 cataract operations during higher specialist training, ${ }^{4}$ no studies have described the number of cases actually being performed by trainees, nor have factors influencing surgical numbers been investigated.

There are many reports from around the world of cataract surgery complication rates for trainees in the initial phase of learning phacoemulsification. $^{5-9}$ Similar reports also exist for experienced surgeons and for overall
Department of

Ophthalmology, St James's University Hospital, Leeds, UK

Correspondence: JC Buchan, Ophthalmology, St James's University Hospital,

Beckett Street, Leeds LS9 7TF, UK

Tel: + 01132433144

Fax: 01132066044.

E-mail: johnbuchan@ gmail.com

Received: 25 March 2008 Accepted in revised form: 23 April 2008 Published online: 12 September 2008

Financial interest: None Department of 
surgical programmes, including surgeons of varied levels of experience. ${ }^{10-12}$ There has, however, been no report of the complication rates of more experienced trainees and as such no suitable benchmark exists for cataract surgical audit by individual trainee surgeons themselves, or by those charged with their ongoing training.

This study provides a description of the surgical experience gained by SpRs in one training region of the UK and the factors influencing this. Rates of posterior capsule rupture (PCR) encountered by those trainees are also reported, providing a much needed comparator for surgeons or trainers in any health-care system wishing to audit the results of more experienced trainees.

\section{Materials and methods}

SpR training is structured into discreet 6-month posts, which cover the breadth of the subspecialities, often rotating through many different hospitals within a defined geographic region. The Yorkshire region has seven hospitals through which ophthalmic SpRs rotate; four teaching hospitals and three district general hospitals. Data were collected from trainees in the Yorkshire region. Any SpR who had been on the Yorkshire SpR training rotation for 6 months or longer (including those in substantive locum posts) was deemed eligible for inclusion, as were any $\mathrm{SpR}$ who had completed their training within the past 6 months. Twenty-eight individuals were identified as eligible; data recruitment was completed between December 2006 and March 2007.

Number of cataract operations and episodes of PCR in each calendar month were recorded from surgical logbooks of all respondents. This process was aided by the high level of uptake of the RCOphth electronic surgical logbook which standardised data recording. ${ }^{13}$

For each completed calendar month reported, note was made of the subspeciality of the post, the gender of the trainee, and their year of training. Each completed calendar month was therefore taken as a discreet unit for the analysis. All research questions were determined before commencement of data collection. Statistical analysis was conducted using SPSS 13.0. Data were tested for approximation to normal distribution before analysis. One sample Student's T-Test was used for calculation of $95 \%$ confidence intervals (95\% CI); Student's T-Test (2-tailed) was also used for comparison of means.

\section{Results}

Of the 28 SpRs identified as meeting the inclusion criteria, data were received from $19 \mathrm{SpRs}$. Of the nine non-responders; five were no longer in the region, being either in fellowship, research, or consultant posts; one surgeon was unable to access the data from their logbook in a usable form for this study; the other three were in their final year of training and although two out of three expressed the desire to contribute, they could not find time to process and submit their data. The 19 responders, 9 female and 10 male, reported having completed a median of 125 cataract operations during their basic surgical training.

\section{Surgical opportunity}

Data from 475 completed calendar months were collected from 19 trainees (mean 25 months contributed per trainee). A total of 4322 cataract operations were reported; a mean of 9.1 (95\% CI 8.6-9.6) operations per month (range 0-33) (Table 1).

District general hospitals (88 months) provided greater exposure to cataract surgery than the teaching hospitals (364 months); mean 10.9 vs 8.5 cases per month $(P=0.005)$. Twenty-three of the months for which data were collected had been from posts outside of Yorkshire, either at the commencement of SpR training before transfer of region or later due to travel for out of programme experience of subspecialist fellowships or research.

Female trainees (240 months) completed mean 9.6 cataract procedures per month compared to males (235 months) with mean 8.6; the difference in the means was not statistically significant $(P=0.055)$.

Trainees therefore performed a mean of 55 cataract operations per 6-month post. The number of cases, however, varied considerably between the different subspecialities with the lowest volume of cataract surgery being found on oculoplastic and paediatric firms with 6.7 and 7.0 mean cataract operations per month being undertaken respectively compared to 12.8 cases per month on general firms (Table 2).

\section{PCR rate}

Ninety-nine episodes of PCR were reported from the 4322 cataract operations. Thus, the overall PCR rate was $2.29 \%$ (95\% CI $2.25-2.33 \%$ ); the range between individual trainees was 3/550 (0.6\%)-9/113 (6.9\%) (Table 1).

Data were grouped according to the year of training in which the surgery was performed. PCR rate was found to have a significant, negative correlation with year of training (Spearman's $\rho=-0.162, P<0.01$ ) as displayed in Table 3.

There was a significant but weak negative correlation between PCR rate and the number of cataract operations undertaken in each 6-month post (Spearman's 
Table 1 Yorkshire SpR's cataract surgical numbers and episodes of PCR

\begin{tabular}{lcccc}
\hline SpR & Cataracts extracted & Length of training (months) & Number of cases of PCR & PCR rate (\%) \\
\hline 1 & 376 & 42 & 8 & 7 \\
2 & 283 & 33 & 6 & 2.1 \\
3 & 235 & 27 & 5 & 2.5 \\
4 & 351 & 35 & 9 & 1.4 \\
5 & 131 & 16 & 15 & 6.9 \\
6 & 446 & 47 & 3 & 3.4 \\
7 & 107 & 9 & 2 & 2.8 \\
8 & 80 & 10 & 2 & 2.5 \\
9 & 139 & 11 & 1 & 1.4 \\
10 & 62 & 7 & 5 & 1.6 \\
11 & 195 & 26 & 5 & 2.6 \\
12 & 236 & 23 & 3 & 2.1 \\
13 & 550 & 44 & 1 & 0.6 \\
14 & 71 & 12 & 7 & 1.4 \\
15 & 333 & 42 & 4 & 2.1 \\
16 & 115 & 15 & 8 & 3.5 \\
17 & 205 & 28 & 1 & 3.9 \\
18 & 290 & 30 & & 2.4 \\
Overall & 117 & 18 & 99 & 0.9 \\
\hline
\end{tabular}

Table 2 Number of cataract operations performed by trainees divided by subspeciality

\begin{tabular}{lcc}
\hline Subspeciality & $\mathrm{n}$ (months) & $\begin{array}{c}\text { Mean cataracts } \\
\text { per month }\end{array}$ \\
\hline Oculoplastics & 53 & 6.7 \\
Paediatric & 118 & 7.0 \\
Medical retina & 63 & 9.0 \\
Vitreo-retinal & 74 & 9.6 \\
Cornea & 55 & 9.8 \\
Glaucoma & 39 & 11.0 \\
Neuro-ophthalmology/uveitis & 22 & 11.0 \\
General & 51 & 12.8 \\
Total & $\mathbf{4 7 5}$ & $\mathbf{9 . 1}$ \\
\hline
\end{tabular}

Table 3 Surgical opportunity and median PCR rate in each year of training

\begin{tabular}{lccc}
\hline Year of training & $\begin{array}{c}\mathrm{n} \\
\text { (months) }\end{array}$ & $\begin{array}{c}\text { Mean cataracts } \\
\text { per month }\end{array}$ & $\begin{array}{c}\text { PCR rate } \\
(\%)\end{array}$ \\
\hline 1st & 217 & 9.2 & 2.5 \\
2nd & 144 & 9.2 & 1.4 \\
3rd & 82 & 10.0 & 1.3 \\
4th/5th & 32 & 5.8 & 2.0 \\
Overall & 475 & $\mathbf{9 . 1}$ & $\mathbf{2 . 1}$ \\
\hline
\end{tabular}

$\rho=-0.084, P<0.01)$, suggesting that a higher volume of surgery leads to lower PCR rate.

\section{Discussion}

Whether or not the UK surgical training structure maintains this two-tier system in the future, the results of this study will remain pertinent in considering the situation and standards expected in the final 4 or 5 years of training, and in analogous structures in other healthcare systems where these exist.

Generation of large volumes of data regarding intraoperative complications will, in practice, rely inevitably on the integrity and record keeping of the surgeons themselves for the quality of the data. This potential problem was not felt to be avoidable by prospective data collection or reference to electronic patient record. Such prospective methods may even exacerbate underreporting of complications where the suggestion of audit by a third party is perceived to exist. The trainee's own logbook is not accessible by anyone other than the trainee and is not used for audit purposes by any external party; motivation to under-report complications is thus minimised by this opportunistic data collection.

As all non-responders were at or beyond the end of SpR training and those who gave a reason for nonresponse cited the plausible explanation of excessive workload, the risk of bias being introduced by potential self-exclusion to hide poor results was felt to be low.

\section{Surgical opportunity}

The mean of over nine cataract operations per month is not adjusted for annual leave or study leave, which together could total over 8 weeks per year, hence the concentration of surgery enjoyed by trainees is greater than this figure suggests. With this level of surgical opportunity, around 500 cataract operations are completed by the end of 4.5 years SpR training; an 
enviable total for any industrialised world ophthalmic training programme.

A previous questionnaire based survey of UK ophthalmologists in basic surgical training found that more cataract surgery was provided in teaching hospitals as compared to district general hospitals. ${ }^{2}$ In our study of higher surgical trainees the situation is reversed with over $25 \%$ more cases provided by posts in district general hospitals. This is likely to be a reflection of the increased focus on subspecialist surgery in teaching hospitals, but will be compounded by the increased opportunities rightly provided for basic trainees in teaching hospitals. Any negative effect on SpRs resulting from the loss of cataract surgical numbers due to the presence of basic surgical trainees is compensated for by the provision of the valuable opportunity to teach junior surgeons.

The same study of basic surgical training also reported that female trainees were given less surgical opportunity than males. ${ }^{2}$ The reasons for this disparity in the early years of training are unclear, as are the reasons for the trend to reversal of this situation reported here. Our study provides welcome evidence that any previously tolerated prejudices, which may have limited opportunities of female surgeons, are not found to persist in ophthalmic higher surgical training in the UK.

Paediatric and oculo-plastic attachments offered the least cataract surgery (Table 2), presumably due to more specialised surgery on offer. Vitreo-retinal and glaucoma attachments might also, in the past, have been expected to focus training on specialist surgery leading to a reduction in cataract numbers. Recent years, however, have seen a trend towards these areas becoming increasingly the exclusive domain of subspecialists; trainees may therefore no longer have opportunity to perform procedures such as trabeculectomy or external retinal detachment repairs, hence the cataract numbers on these attachments are maintained.

\section{PCR}

The learning curve in phacoemulsification has been well demonstrated previously, even for experienced surgeons. ${ }^{12}$ The trend to reduction in PCR through the training programme is unsurprising, and the increase in complication rate from the data from trainees' 4 th $/ 5$ th year of training is explicable by their reduced volume of cataract operations (Table 3) combined with the presumed tendency to take on more difficult cases that would have been avoided earlier in training.

\section{Audit benchmark}

The published results of residency programmes in the United States of America may be proposed as an existing potential source of benchmark for auditing UK trainees. However, our result of $2.3 \%$ compares extremely favourably with these, as one US reviewer in 2003 estimated an overall rate of $7 \%$ vitreous loss from the combined published studies of residents' cataract surgery, ${ }^{5}$ although all these reports included far fewer cases and fewer surgeons than our study. $5,6,8,14-16$

Comparison of higher specialist training programmes in the UK with US residency programmes is not particularly valid due to the higher level of experience of UK SpRs compared to the US residents. The minimum average number of cataract operations that a residency programme must provide to gain accreditation is 45 (defined by the Residency Review Committee responsible for accrediting residency programmes), even if the actual average cataract numbers of surgeries performed in US residency programmes is more than double this figure. ${ }^{17}$

It is generally accepted that the UK National Cataract Surgery Survey ${ }^{11}$ published in 1999, which found a PCR with vitreous loss rate for phacoemulsification of $4.4 \%$, is no longer an acceptable benchmark for audit of surgical complications. With the evolution of the machines, the techniques, and the surgeons themselves, better results should now be expected. Other benchmarks for audit must therefore be sought.

A series of 1000 cataract procedures performed on consultant supervised training lists reported a PCR rate of $1.1 \%$, but included a mixture of consultant, SpRs, and basic surgical trainees, and no subgroup analysis was offered. ${ }^{10}$ This may be a useful figure for audit in a similar training programme, but would not be useful to individual SpRs or to a training programme director wishing to evaluate their own higher specialist trainees. Other potential benchmarks from the RCOphth Guidelines for Cataract Surgery 2004 (amended 2007) would be the UK Electronic Patient Record Audit (2002/3) with a zonular rupture/PCR rate of $2.68 \%$, but again this figure is derived from an overall surgical service.

Our study therefore offers this figure of $2.3 \%$ as a useful benchmark for individual SpRs and directors of training alike. As a mean, it represents the PCR rate for a trainee of 25-months experience, and will therefore underestimate the risk for junior SpRs and overestimate that for their more experienced colleagues, but would provide a good direct comparator for evaluation of the risk conveyed to patients in a region by a cohort of higher surgical trainees amalgamating their collective data by the same method employed in this study. Such a process of audit at the training programme level is not regularly conducted, and this may represent a missed opportunity to reduce surgical risk by specific training interventions or identification of local risk factors for increased 
complication, such as low volume of surgery or older phacoemulsification machines in particular posts.

\section{Summary}

Ophthalmic trainees in the UK can expect a good level of exposure to cataract surgery during their training with over 50 cases per 6-month post being undertaken by SpRs in the Yorkshire region. There are posts within the training scheme offering significantly less cataract surgery than others, and these posts should perhaps be avoided by trainees struggling with surgery towards the start of their training.

With in excess of 300000 cataract procedures now performed annually in the UK (www.hesonline.nhs.uk/), attention will need to be paid to quality assurance within the training process to meet the challenges of service provision in the future. Both the trainees themselves and those charged with overseeing their training have a responsibility to the patients involved to ensure that problems are identified and remedial measures taken where increased levels of complication are being experienced by individuals or by a cohort of trainees when considered as a group. This study provides a much-needed benchmark for PCR of $2.3 \%$ for higher specialist trainees in the UK, and those of equivalent experience in other systems.

\section{References}

1 Acheson J. Are ophthalmologists overtrained? $\mathrm{Br} \mathrm{J}$ Ophthalmol 2001; 85(4): 383-384.

2 Gibson A, Boulton MG, Watson MP, Moseley MJ, Murray PI, Fielder AR. The first cut is the deepest: basic surgical training in ophthalmology. Eye 2005; 19(12): 1264-1270.

3 Watson MP, Boulton MG, Gibson A, Murray PI, Moseley MJ, Fielder AR. The state of basic surgical training in the UK: ophthalmology as a case example. J R Soc Med 2004; 97(4): 174-178.

4 Curriculum of higher specialist training in ophthalmology. Royal College of Ophthalmologists, 2003; CH03/03, p 12.
5 Quillen DA, Phipps SJ. Visual outcomes and incidence of vitreous loss for residents performing phacoemulsification without prior planned extracapsular cataract extraction experience. Am J Ophthalmol 2003; 135(5): 732-733.

6 Bhagat NM, Nissirios NM, Potdevin LB, Chung J, Langer P, Zarbin $\mathrm{M}$ et al. Resident-performed phacoemulsification cataract surgery at New Jersey medical school. $\mathrm{Br} J$ Ophthalmol 2007; 91(10): 1315-1317.

7 Thomas R, Naveen S, Jacob A, Braganza A. Visual outcome and complications of residents learning phacoemulsification. Indian J Ophthalmol 1997; 45(4): 215-219.

8 Blomquist PH, Rugwani RM. Visual outcomes after vitreous loss during cataract surgery performed by residents. J Cataract Refract Surg 2002; 28(5): 847-852.

9 Tayanithi P, Pungpapong K, Siramput P. Vitreous loss during phacoemulsification learning curve performed by third-year residents. J Med Assoc Thai 2005; 88(Suppl 9): S89-S93.

10 Zaidi FH, Corbett MC, Burton BJ, Bloom PA. Raising the benchmark for the 21st century - the 1000 cataract operations audit and survey: outcomes, consultantsupervised training and sourcing NHS choice. $\mathrm{Br} \mathrm{J}$ Ophthalmol 2007; 91(6): 731-736.

11 Desai P, Minassian DC, Reidy A. National cataract surgery survey 1997-8: a report of the results of the clinical outcomes. Br J Ophthalmol 1999; 83(12): 1336-1340.

12 Martin KR, Burton RL. The phacoemulsification learning curve: per-operative complications in the first 3000 cases of an experienced surgeon. Eye 2000; 14(Part 2): 190-195.

13 http://www.rcophth.ac.uk/training/logbook.

14 Allinson RW, Metrikin DC, Fante RG. Incidence of vitreous loss among third-year residents performing phacoemulsification. Ophthalmology 1992; 99(5): 726-730.

15 Corey RP, Olson RJ. Surgical outcomes of cataract extractions performed by residents using phacoemulsification. J Cataract Refract Surg 1998; 24(1): 66-72.

16 Tarbet KJ, Mamalis N, Theurer J, Jones BD, Olson RJ. Complications and results of phacoemulsification performed by residents. J Cataract Refract Surg 1995; 21(6): 661-665.

17 Binenbaum G, Volpe NJ. Ophthalmology resident surgical competency: a national survey. Ophthalmology 2006; 113(7): 1237-1244. 